\title{
Prevalência de transtornos mentais menores em estudantes universitários $^{1}$
}

\author{
Ednéia Albino Nunes Cerchiari \\ Universidade Estadual de Mato Grosso do Sul \\ Dorgival Caetano \\ Universidade Estadual de Campinas \\ Odival Faccenda \\ Universidade Estadual de Mato Grosso do Sul
}

\begin{abstract}
Resumo
O presente estudo é parte da pesquisa de doutorado que teve por objetivo estimar a prevalência de Transtornos Mentais Menores (TMM) na população de estudantes universitários dos cursos de Ciência da Computação, Direito, Letras e Enfermagem, da Universidade Estadual de Mato Grosso do Sul e do curso de Enfermagem da Universidade Federal de Mato Grosso do Sul, como também verificar a associação de algumas variáveis sociais e acadêmicas com os TMM. O estudo incluiu todos os estudantes dos referidos cursos, matriculados no primeiro semestre de 2002, e foi do tipo corte transversal. A pesquisa contou com uma amostra de 558 sujeitos, sendo 396 (71\%) do sexo feminino e 161 (29\%) do sexo masculino. Para coleta dos dados utilizaram-se dois questionários auto-aplicáveis: QSG-60 e QDSD. Constatou-se uma prevalência de 25\% de TMM entre os estudantes, destacando-se, como transtorno principal, os distúrbios psicossomáticos.
\end{abstract}

Palavras-chave: distúrbios psicossomáticos; ansiedade; depressão; distúrbios do sono

\begin{abstract}
Prevalence of minor mental disorders in undergraduate students. The present study had as its main objective to estimate the prevalence of Minor Mental Disorders (TMM) in students enrolled in the courses offered at State University of Mato Grosso do Sul (Computer Science, Law, Arts, and Nursing), and at Federal University of Mato Grosso do Sul (Nursing) to investigate the relationship of MMD with both sociodemographic variables and learning process. This was a transversal study that included all students of all years in each course, enrolled in the first semester of 2002. The sample was composed of 558 students: 396 (71\%) females and 161 (29\%) males. Two self-report questionnaires (QSG-60 and QDSD) were used for collection of data. A prevalence of $25 \%$ of MMT was found among the students and the main disorder was psychosomatic disturbance.
\end{abstract}

Keywords: psychosomatic disturbance; anxiety; depression; sleep disturbance

$\mathrm{O}$ s estudos em epidemiologia psiquiátrica têm sido, em sua maioria, realizados nos países ocidentais e os resultados indicam que $90 \%$ da morbidade psiquiátrica encontrada refere-se a distúrbios não-psicóticos (Goldberg \& Huxley, 1992). Porém, devido às várias questões conceituais e metodológicas imbricadas no diagnóstico dos distúrbios não-psicóticos, especialmente as que se referem à distinção entre depressão e ansiedade, alguns estudos têm utilizado categorias diagnósticas mais amplas, designando-as por morbidade psiquiátrica menor (MPM; Mari, 1987), transtornos mentais comuns (TMC; Coutinho, 1995; Facundes, 2002; Facundes \& Ludermir, 2005; Ludermir, 2000) e problemas psiquiátricos menores (PPM; Benvegnú, Deitos, \& Copette, 1996).
Essas terminologias referem-se a sintomas ansiosos, depressivos e somatoformes, com elevada prevalência na população geral adulta e diagnosticados em pelo menos um terço dos pacientes de atendimento em serviços primários de saúde (Coutinho, 1995). Neste estudo, a terminologia empregada é a de transtornos mentais menores (TMM). Vários estudos, citados por Coutinho (1995) mostram prevalências de TMM variando de $7 \%$ a $26 \%$, com uma média de $17 \%$, sendo $12,5 \%$ entre os homens e $20 \%$ entre as mulheres.

No Brasil, Almeida Filho et al. (1992) conduziram um "Estudo multicêntrico de morbidade psiquiátrica em áreas metropolitanas”, numa amostra de casas aleatoriamente selecionadas nas cidades de Brasília, São Paulo e Porto Ale- 
gre, com o objetivo de estimar as prevalências globais de distúrbios psiquiátricos (EPG) na população e as prevalências de demanda potencial (DPE) de serviços destinados ao tratamento de distúrbios psiquiátricos. A análise dos grupos diagnósticos revelou que os distúrbios neuróticos, especialmente ansiedade e fobias, são, em termos numéricos, os principais problemas de saúde mental da população urbana, com EPG variando de $8 \%$ a $18 \%$ e estimativas de DPE de $5 \%$ a $12 \%$; as depressões não-psicóticas afetam particularmente o sexo feminino, com prevalências de até $14 \%$, enquanto que o alcoolismo acomete principalmente o sexo masculino (11 homens: 1 mulher), atingindo prevalências globais e estimativas de demanda de até $9 \%$.

Rimmer, Halikas e Schuckit (1982), em estudos relativos a estudantes universitários, detectaram uma prevalência de $14 \%$ a $19 \%$ de problemas surgidos em algum momento da vida acadêmica, em que um quarto dos alunos diagnosticados com algum tipo de doença psiquiátrica procurou atendimento, sendo que a depressão manifestou-se como distúrbio predominante. Niemi (1988), na Finlândia, em um levantamento do perfil da clientela atendida no serviço de saúde universitário, concluiu que ansiedade e medos constituíram 35\% dos sintomas; depressão e solidão, $21 \%$ e dificuldades nas relações sociais, 18\%. Demonstrou, ainda, que a freqüência de distúrbios psiquiátricos na população universitária variou de $6 \%$ a $29 \%$.

No Brasil, além da escassez de estudos epidemiológicos sobre a morbidade psiquiátrica em estudantes universitários, há carência de rigor metodológico e estatístico. Os estudos nacionais publicados no período de 1958 a 2002 podem ser classificados em três grupos.

Levantamento dos índices de utilização dos serviços em saúde mental (Abdo, 1987; Abdo \& Fortes, 1978; Fernandez \& Rodrigues, 1993; Fortes \& Abdo, 1981; Hahn, 1994; Loreto, 1958, 1963, 1964, 1965a, b, 1972, 1985; Lucena \& Loreto, 1968; Millan, 1997; Noto, Avancine, Martins, \& Zimmermann, 2001).

Estudos Epidemiológicos de problemas específicos utilizando amostras representativas, com as seguintes subdivisões e autores: (a) distúrbios não psicóticos (Benvegnú et al., 1996; Facundes, 2002; Facundes \& Ludermir, 2005; Giglio, 1976, 1981); (b) abuso de anfetaminas (Cavalcanti, 1958; Ribas, 1975); (c) suicídio, pensamento suicida e tentativas de suicídio (Milan, 1997; Miranda \& Queiroz, 1991); (d) uso de bebidas alcoólicas (Borini, Oliveira, Martins, \& Guimarães, 1994); (e) processo ensino-aprendizagem e saúde mental do estudante de medicina (Cianflone \& Fernandez, 1993).

O terceiro grupo abrange um número relativamente grande de estudos com características metodológicas especiais. São eles: (a) considerações (Albuquerque, 1973; D’Andrea, 1984; Fortes, 1972; Millan, Souza, De Marco, Rossi, \& Arruda, 1998); (b) levantamento do interesse de universitários em cursos de saúde (Almeida, Souza, Paiva, \& Lima, 1980); (c) estimativa de incidência de crises em estudantes de medicina ( $\mathrm{D}^{\prime}$ Andrea \& Almeida, 1988); (d) levantamento dos tipos de serviços de apoio ao estudante de enfermagem (Jorge \& Rodrigues, 1995); (e) levantamento das necessidades do estudante de enfermagem (Jor- ge, 1996; Rodrigues, Scatena, \& Labate, 1997); (f) revisão histórica (Hahn, Ferraz, \& Giglio, 1999).

A grande maioria dos estudos está relacionada aos levantamentos dos índices de utilização dos serviços de saúde mental oferecidos pelas universidades. Estudos epidemiológicos de TMM na população estudantil universitária são em pequeno número e lidam com populações específicas de estudantes de Medicina (Benvegnú et al. 1996); Medicina, Enfermagem, Educação Física e Odontologia (Facundes, 2002; Facundes \& Ludermir, 2005) e apenas dois nas outras áreas do conhecimento: Ciências Tecnológicas, Exatas, Biológicas e Humanas (Giglio, 1976; Lucena et al., 1957, citado por Loreto, 1958).

O presente estudo é parte do Projeto de Pesquisa em Saúde Mental e Qualidade de Vida em Estudantes Universitários, aprovado pelo Comitê de Ética em Pesquisa da Faculdade de Ciências Médicas da Universidade Estadual de Campinas (UNICAMP). A pesquisa teve por objetivo estimar a prevalência de transtornos mentais menores (TMM), bem como verificar a associação de algumas variáveis sociais e acadêmicas com os TMM em estudantes universitários.

\section{Método}

Na presente investigação foi utilizado o corte transversal analítico como desenho de estudo, cujos dados foram coletados mediante aplicação de dois questionários. A população de estudo foi definida como os alunos, de ambos os sexos, matriculados nos cursos que tinham seriação completa na Universidade Estadual de Mato Grosso do Sul, unidade de Dourados, e no curso de Enfermagem da Universidade Federal de Mato Grosso do Sul, campus de Campo Grande.

Os questionários foram aplicados em sala de aula, em um estudo piloto, com treze acadêmicos sorteados aleatoriamente dos seguintes cursos: Letras (4), Enfermagem (4) e Direito (5), um aluno de cada série de cada curso, a fim de testar clareza, objetividade, tempo de aplicação, simplicidade e facilidade de resposta, receptividade dos alunos à pesquisa e, ainda, como parte do treinamento dos auxiliares da pesquisa.

Em seguida, os instrumentos foram aplicados em uma amostra de 558 estudantes de duas universidades públicas: (1) unidade de Dourados, da Universidade Estadual do Mato Grosso do Sul, nos cursos de Ciência da Computação, Direito, Letras, Enfermagem; (2) Campus de Campo Grande da Universidade Federal do Mato Grosso do Sul, no curso de Enfermagem. Estas amostragens foram feitas em duas datas previamente determinadas, de acordo com a especificidade de cada curso. Entre os 624 estudantes universitários matriculados nos cursos pesquisados, em junho e julho de 2002, não foram contados os que obtiveram zero em todas as disciplinas e aqueles que não compareceram em nenhuma, bem como os cancelamentos. Desse total (624), compareceram 561 alunos ao local da pesquisa. Após terem lido o Termo de Consentimento Livre e Esclarecido, três alunos se retiraram da sala de aula. Os demais (558; 89,4\%) responderam ao questionário, de acordo com os princípios éticos contidos na De- 
claração de Helsinque (1996), da World Medical Association e do Ministério da Saúde do Brasil para pesquisas envolvendo seres humanos.

As variáveis respostas utilizadas no estudo foram o escore total geral e os escores de cada um dos cinco fatores do Questionário de Saúde Geral com sessenta itens (QSG-60; Goldberg, 1972) adaptado à nossa população, por Pasquali, Gouveia, Andriola, Miranda \& Ramos (1996).

O Questionário de Saúde Geral (QSG) é um instrumento de rastreamento, auto-aplicável, simples, confiável, destinado a identificar distúrbios menores em saúde mental (depressão, ansiedade, disfunção social e sintomas somáticos), o qual avalia se um determinado sentimento percebido pelo sujeito no presente é diferente de seu sentimento usual. Focaliza a habilidade do sujeito de identificar as funções normais e o surgimento de qualquer distúrbio. É ideal para ser usado na comunidade e em ambientes não psiquiátricos. É constituído por cinco fatores: (1) tensão ou stress psíquico; (2) desejo de morte; (3) falta de confiança na capacidade de desempenho/auto-eficácia; (4) distúrbios do sono; (5) distúrbios psicossomáticos e o fator geral que verifica a severidade da ausência de saúde mental. Cada fator é composto por um conjunto de itens que foram determinados por possuir uma carga fatorial igual ou superior a +/- 0,30 na matriz padrão e congruência semântica com os demais itens do fator. Os itens consistem de questões que procuram saber se a pessoa experimentou recentemente um determinado sintoma ou comportamento numa escala de 4 pontos, variando de 1 (não absolutamente) a 4 (muito mais do que de costume) sendo que, uma parte dos itens expressa os sintomas diretamente e outra parte expressa o comportamento normal. Os escores dos respondentes nos cinco fatores específicos e no geral são obtidos pelo somatório das respostas aos itens específicos de cada fator, segundo o sistema Likert de escalagem (1, 2, 3, e 4), em que 1 representa a ausência do sintoma enunciado pelo item e 4 representa a presença forte do mesmo sintoma. O TMM foi definido por escores acima do percentil noventa, tomando-se como referência os valores apresentados por Pasquali et al. (1996), que se baseia na verificação de que, em geral, há cerca de10\% de casos psiquiátricos numa população normal.

As variáveis de controle (aspectos sociais e acadêmicos) utilizadas neste estudo foram: gênero, faixa etária, estado civil, procedência, tipo de moradia, renda familiar, tempo de locomoção, atividade remunerada, ano de ingresso na universidade, curso, período, série e opção de escolha do curso, obtidas pelo Questionário de Dados SócioDemográficos.

O processamento e análise dos dados foram realizados através do software SPSS, utilizando-se o coeficiente de correlação de Spearman para verificar se o grau de associação entre as variáveis foi significativamente diferente de zero. Utilizou-se ANOVA para fazer análise de variância e o Teste de Tukey para fazer comparações múltiplas entre os níveis das variáveis de controle. Além disso, foi realizada uma regressão linear múltipla stepwise para determinar quais variáveis permaneceriam no modelo. O nível estabelecido para verificar significância estatística foi de 5\%.

\section{Resultados}

O perfil sócio-demográfico dos estudantes universitários nos cursos investigados mostrou predominância do sexo feminino (71\%), sendo de $84 \%$ no curso de Letras, $83 \%$ no de Enfermagem e 57\% no de Direito. No curso de Computação predominou o sexo masculino (79\%). Tiveram maiores freqüências: a faixa etária entre 19 e 24 anos (75\%), os solteiros (80\%), residentes no município-sede da universidade e procedentes da região urbana (53\%), sem atividade remunerada (61\%), renda familiar mensal entre 0 a $\mathrm{R} \$ 1.000,00$ (39\%), residindo com a família (61\%), o curso que freqüentam foi objeto de sua primeira escolha (58\%) e estudam em tempo integral (46\%).

Na Tabela 1 são apresentados os valores relativos à prevalência do perfil sintomático da amostra total e por curso, tomando como referência os valores apresentados por Pasquali et al. (1996) (percentil acima de 90\%). Observou-se que a prevalência de TMM foi de $25 \%$ e os fatores mais acentuados na amostra total foram: distúrbios psicossomáticos (29\%), estresse psíquico (28\%) e desconfiança no desempenho (26\%), respectivamente por ordem de magnitude. Dentre os cursos, o de Enfermagem apresentou a prevalência total mais elevada (34\%), seguindo-se o de Letras (22\%), o de Direito (17\%) e, por fim, o de Ciência da Computação (9,0\%), destacando-se os seguintes problemas por cursos: Enfermagem - estresse psíquico, distúrbios psicossomáticos e desconfiança no desempenho; Letras e Direito - distúrbios psicossomáticos, estresse psíquico e desconfiança no desempenho respectivamente; Ciência da Computação - desconfiança no desempenho.

Tabela 1

Prevalência do perfil sintomático, por fatores e cursos, obtido através do percentil 90, tomando como referência os valores de Pasquali et al. (1996)

\begin{tabular}{lccccc}
\hline Curso & C. Computação & Direito & Letras & Enfermagem & Total \\
\hline Fatores & $n=73$ & $n=159$ & $n=69$ & $n=257$ & $N=558$ \\
G - Ausência de saúde mental & $9 \%$ & $17 \%$ & $22 \%$ & $34 \%$ & $25 \%$ \\
1 - Estresse psíquico & $9 \%$ & $17 \%$ & $26 \%$ & $41 \%$ & $28 \%$ \\
2 - Desejo de morte & $4 \%$ & $13 \%$ & $19 \%$ & $18 \%$ & $15 \%$ \\
3 - Desconfiança no desempenho & $18 \%$ & $23 \%$ & $19 \%$ & $33 \%$ & $26 \%$ \\
4 - Distúrbios do sono & $4 \%$ & $13 \%$ & $18 \%$ & $27 \%$ & $19 \%$ \\
5 - Distúrbios psicossomáticos & $10 \%$ & $19 \%$ & $27 \%$ & $40 \%$ & $29 \%$ \\
\hline
\end{tabular}


As variáveis de controle (gênero, faixa etária, estado civil, procedência, tipo de moradia, atividade remunerada, renda familiar, ano de ingresso na universidade, curso, período, série, opção de escolha do curso) foram correlacionadas, individualmente, com TMM. Dessas, local, idade, estado civil, procedência, renda familiar e tempo de deslocamento não apresentaram correlações significativas $(p>0,05)$ com nenhum dos fatores do QSG-60 e foram excluídas da análise de regressão. Gênero, ano de ingresso, curso, período, série, atividade remunerada, opção de escolha do curso e tipo de moradia $(p<0,05)$ foram incluídas na regressão linear múltipla stepwise como variáveis independentes e TMM como a dependente. As variáveis gênero, ano de ingresso, atividade remunerada, curso e tipo de moradia se correlacionaram significativamente $(p<0,05)$ com os TMM.

Em relação à variável ano de ingresso na universidade, tanto no Fator geral (ausência de saúde mental) quanto no Fator 1 (tensão ou estresse psíquico) os alunos que ingressaram há um ano apresentaram escores significativamente menores em comparação com aqueles que estão de 2 a 5 anos. Similar tendência ocorreu no Fator 5 (distúrbios psicossomáticos), no qual alunos há um ano na Universidade apresentam escores significativamente menores do que aqueles que estão há 3 e os que estão há 5 anos (Tabela 2). No Fator 2 (desejo de morte), todos os anos apresentaram escores médios relativamente baixos (comparados com os valores de referência; Pasqualli et al., 1996) e sem diferença significativa entre eles $(p>0,05)$. Por outro lado, no Fator 3 (falta de confiança na capacidade de desempenho/auto-eficácia), os estudantes de todos os anos apresentaram escores médios significativamente maiores do que aqueles que estão apenas há um ano ( $\mathrm{p}<0,05)$. Já no fator 4 (distúrbios do sono) não houve diferença significativa entre os grupos estudados $(p=0,07)$. Em geral, na medida em que o acadêmico permanece na universidade há um aumento da tensão ou estresse psíquico, distúrbios psicossomáticos, falta de confiança na capacidade de desempenho e auto-eficácia, indicando assim uma diminuição da saúde mental geral.

A falta de uma atividade remunerada interfere negativamente na saúde mental geral bem como nos demais fatores (Tabela 3), haja vista que os estudantes que se encontravam nessa situação apresentaram escores significativamente maiores dos apresentados por aqueles estudantes que estavam empregados ou realizando estágios remunerados $(p<0,05)$.

Nos fatores: Fator Geral (ausência de saúde mental), Fator 1 (tensão ou estresse psíquico), Fator 3 (desconfiança no próprio desempenho), Fator 4 (distúrbios do sono) e Fator 5 (distúrbios psicossomáticos), os alunos do Curso de Enfermagem apresentaram escore médio significativamente maior, quando comparados com o apresentado pelo dos outros cursos (Tabela 4). No Fator 2 (desejo de morte), porém, o Curso de Enfermagem apresentou escore superior apenas em relação ao curso de Ciência da Computação $(p<0,05)$.

As mulheres apresentaram escore médio significativamente maior do apresentado pelos homens, tanto no fator de saúde mental geral quanto em todos os outros fatores.
Os estudantes que moram em república e aqueles que moram em pensionatos apresentaram escore médio significativamente maior, respectivamente, em estresse psíquico (Fator 1 ) e em distúrbios do sono (Fator 4$)(p<0,05)$, quando comparados aos que moram com a família.

\section{Discussão e Conclusão}

A prevalência global de TMM em nossa amostra foi de $25 \%$, o que está abaixo daquela referida em outros estudos com universitários (Benvegnú et al., 1996; Facundes, 2002; Facundes \& Ludermir, 2005; Giglio, 1976; Rimmer et al., 1982) os quais encontraram de $31 \%$ a $39 \%$. Entretanto, essas taxas estão bem acima daquelas encontradas em estudos populacionais brasileiros, que variam entre 8\% e 23\% (Almeida Filho et al., 1992 - 8\% a 18\%; Coutinho, 1976 - 22,6\%; Santana $1978-14,5 \%)$.

Na amostra total, os distúrbios psicossomáticos (Fator 5) foram os principais problemas de saúde mental da população estudantil universitária com prevalência de 29\%, seguido por tensão ou estresse psíquico (Fator 1), com 28\%, e falta de confiança na capacidade de desempenho/auto-eficácia (Fator 3), com 26\%. Esse dado permite levantar a hipótese de que a falta de confiança na capacidade de desempenho/auto-eficácia desencadeia tensão ou estresse psíquico; este, por sua vez, se manifesta no corpo (distúrbios psicossomáticos), demonstrando assim a dificuldade dos estudantes em lidar com as emoções em uma situação conflitante, isto é, não conseguem elaborá-las ou representá-las por atos ou palavras, manifestando-as através do corpo. Esses achados contrariam estudos que mostram a depressão como transtorno predominante entre os universitários (Brown, 1978; Grofe, 1963; Kysar, 1962; O’Neil \& Marziali, 1976; Spalt, 1977). Porém, neste estudo, o Fator 2 (desejo de morte), apesar de elevado, obteve o menor escore.

As variáveis sócio-demográficas (estado civil, procedência, renda familiar) não se correlacionaram com TMM, corroborando resultados de estudos nacionais (Benvegnú et al., 1996; Facundes, 2002; Facundes \& Ludermir, 2005). Contudo, contrário aos resultados deste estudo, Benvegnú et al. (1996) relatam que faixa etária apresentou uma associação significativa com os TMM. Por outro lado, a variável com quem mora, que foi significativa neste estudo, é também relatada por Facundes (2002) e Facundes e Ludermir (2005). A variável gênero mostrou-se significativamente correlacionada com os TMM ( $p<0,05)$, contrariando esses mesmos estudos, mas confirmando estudo similar de Giglio (1976), bem como estudos populacionais no Brasil (Aguiar, 1980; Almeida-Filho et al., 1983; Santana, 1982) e no exterior (Dohrenwend \& Dohrenwend, 1980; Lennon, 1987; Paykel, 1991; Rosenfeld, 1989; citados por Almeida Filho et al., 1992).

No sexo feminino predominou o Fator 1 (tensão ou estresse psíquico), o que está em acordo com estudo de Almeida-Filho et al. (1992), enquanto que no sexo masculino predominou o Fator 3, que compreende o construto falta de confiança na capacidade de desempenho/auto-eficácia. 

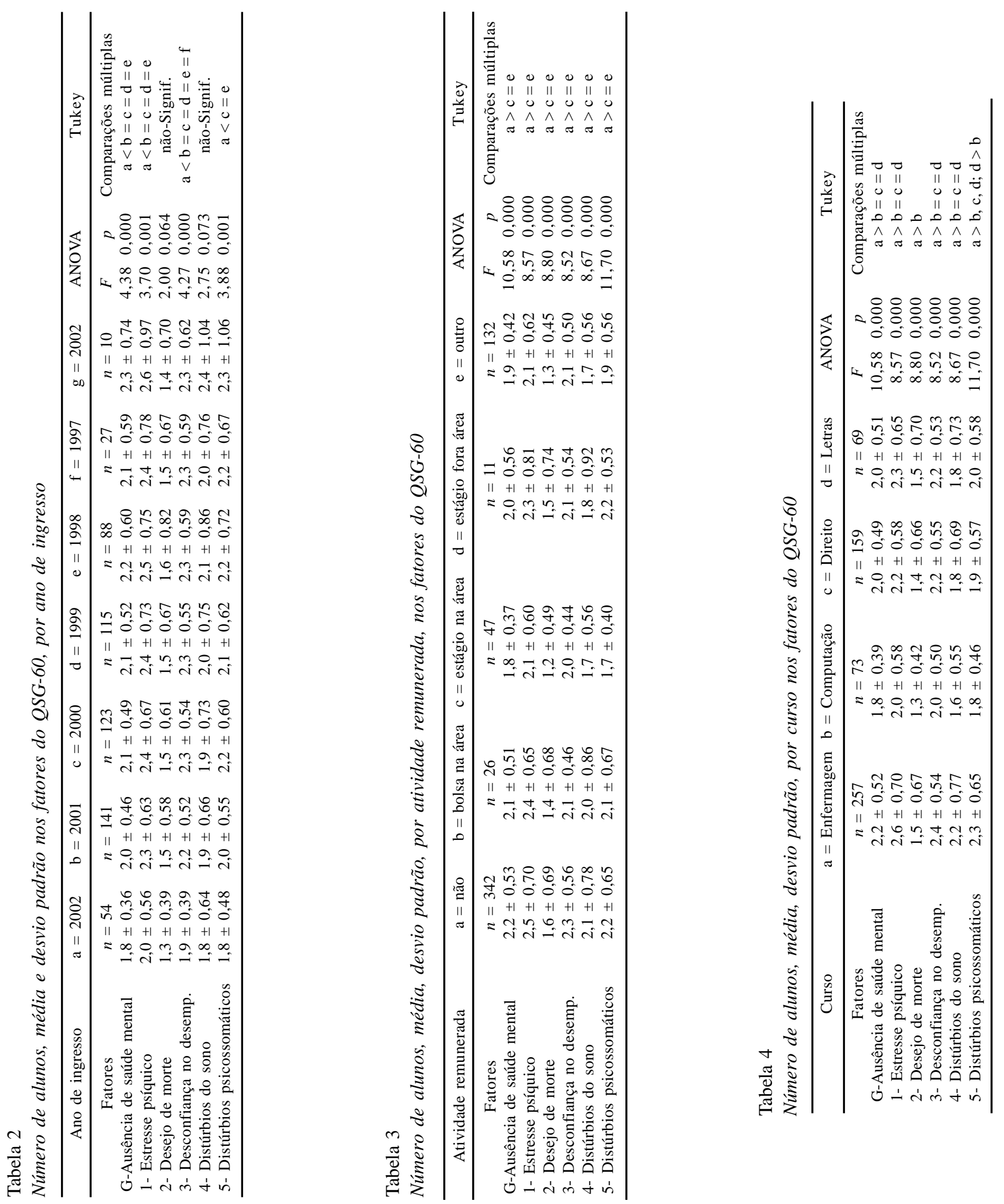
Ao incluirmos a variável ano de ingresso na universidade, partimos da hipótese de que nos anos iniciais (primeiro e segundo anos) os estudantes, em razão da adaptação ao novo modelo de vida imposto pelo mundo universitário, estariam mais suscetíveis a perturbações emocionais. No entanto, os resultados refutaram esta hipótese, pois as dificuldades emocionais, apesar de elevadas, foram significativamente menores no primeiro ano em comparação aos demais. Contudo, estudos realizados por Giglio (1976; 1981), Cianflone \& Fernandez (1993) e Fortes \& Abdo (1981) apontam que geralmente as dificuldades emocionais dos estudantes surgem no final do primeiro ano ou no primeiro semestre do segundo. Esse último autor atribui essas dificuldades ao ingresso de alunos com perturbações incipientes agravadas no decorrer do primeiro ano, ao esforço de adaptação ao novo modelo de vida e a mobilização de disposições mórbidas pré-existentes pela sobrecarga dos alunos que conseguiram ingressar na universidade sem base. Benvegnú et al. (1996), Facundes (2002) e Facundes e Ludermir (2005), por sua vez, não encontraram diferença significativa de prevalência de TMM entre os períodos iniciais (38,9\% e 36,0\%, respectivamente), comparados aos intermediários (25,9\% e 33,0\%), indicando taxas elevadas tanto no período de chegada à Universidade quanto ao longo da trajetória acadêmica.

No presente estudo, o terceiro ano foi o de mais problemas de saúde mental, tais como tensão/estresse psíquico, falta de confiança na capacidade de desempenho/auto-eficácia, distúrbios do sono e distúrbios psicossomáticos.

É interessante destacar que alunos no último ano de seus cursos ( $4^{\underline{0}}$ ano de Ciência da Computação, Enfermagem e Letras e $5^{0}$ ano de Direito) obtiveram escores altos no Fator 5 (falta de confiança na capacidade de desempenho/auto-eficácia), sugerindo sentimento de incapacidade de desempenhar satisfatoriamente suas tarefas diárias como também receios quanto ao futuro. É provável que isso se deva, em alguns casos, mais a conflitos internos individuais, em outros, mais a fatores externos suficientemente fortes, e em outros, a uma combinação desses fatores. Os fatores externos poderiam estar relacionados à universidade e à sociedade, que fornecem ao jovem formando garantias mínimas de realização profissional e pessoal, tornando-o assim inseguro quanto ao mercado de trabalho.

$\mathrm{O}$ fato de que quase $60 \%$ dos alunos fizeram o curso desejando-o, contra apenas 9\% daqueles que o fizeram por falta de alternativa, sugere-se, por um lado, que a universidade não tem preparado devidamente o acadêmico para o exercício profissional, deixando-o inseguro quanto ao futuro. Por outro lado, a estrutura sócio-econômica atual intensifica os sentimentos de insegurança do estudante diante das incertezas do futuro, concordando com Giglio (1976) e Silva (1994, citado por Jorge, 1996), ao indicarem, como um dos fatores agravantes dos sentimentos negativos dos estudantes universitários, a preocupação com o mercado de trabalho.

Os estudantes do curso de Enfermagem apresentam a maior prevalência de TMM (34\%), seguida dos cursos de Letras (22\%), de Direito (17\%) e de Ciência da Computação
(9\%). Os primeiros estão acima daquelas taxas encontradas em estudos populacionais, porém, próximas àquelas obtidas com estudantes da área da saúde: Medicina - 31\% (Benvegnú et al., 1996) e Enfermagem - 31,8\% (Facundes, 2002; Facundes \& Ludermir, 2005). Um alto escore no Fator 1 (estresse psíquico) mostrou que o processo de aprendizado em Enfermagem é estressante, provocando um efeito negativo sobre a performance acadêmica, saúde física e bem estar emocional.

O curso de Letras (22\% de TMM) teve no Fator 5 (distúrbios psicossomáticos, 27\%) seu maior escore, seguido do Fator 2 (desejo de morte, 19\%) o que sugere um funcionamento mental peculiar, em que os distúrbios psicossomáticos e sentimentos depressivos seriam conseqüências de estados de tensão crônica.

O curso de Direito apresentou 17\% de prevalência total de TMM, o que está dentro dos valores encontrados em estudos populacionais internacionais e nacionais. Porém, a prevalência de $23 \%$ no Fator 3 (falta de confiança na capacidade de desempenho/auto-eficácia) está no limite, levandose em conta os estudos populacionais nacionais. Isso sugere ingresso de alunos com perturbações incipientes e/ou processo educativo como agente desencadeador de sofrimento psíquico. Um estudo de Frank (1979) com estudantes de Direito do sexo feminino, indicou que a busca da carreira jurídica era decorrente de "um meio de salvaguardar direitos e privilégios individuais” e que a motivação inconsciente era "uma tentativa positiva de reparar uma auto-estima ferida” (p. 69).

Ciência da Computação apresentou um índice total de TMM (9\%) em todos os fatores, exceto no Fator 3 (falta de confiança na capacidade de desempenho) com 18\%, abaixo, até mesmo, do esperado para a população geral (10\%).

É interessante notar que a ocorrência de sofrimento psíquico tende a ser maior nos cursos em que o objeto de estudo tem maior subjetividade, em especial quando este objeto é o homem e o seu modo de ser, com toda sua complexidade.

Os achados relativos aos tipos de moradia do estudante universitário (com a família, com parentes, sozinho, em república e em pensionato) indicam que morar com a família favorece o bem-estar psicológico do estudante universitário, enquanto que morar em república e pensionato são fatores de risco à saúde mental, o que está em acordo com os estudos de Giglio (1976), Borini et al. (1994) e Hahn (1994).

Os alunos que tinham uma atividade remunerada apresentaram os menores escores no GHQ-60, corroborando estudo de Giglio (1976). Isso desfaz a suposição de que o trabalho concomitante aos estudos é fator de estresse que interfere na produção acadêmica.

Apesar das variáveis escolhidas serem importantes no processo educativo e saúde mental do estudante, estas, no entanto, não esgotam o universo desses fatores. Alguns desses fatores, por exemplo, insatisfação com a universidade e a sua influência na saúde mental do estudante, não foram aprofundados; ficando em aberto a pergunta se a percepção da universidade, como insuficiente e insatisfatória, por parte dos alunos, seria em razão das dificuldades emocionais des- 
ses alunos ou de fato a universidade não estaria correspondendo às expectativas do seu alunado, desencadeando nele distúrbios emocionais. Essas questões naturalmente não exclusivas e atualmente são difíceis de serem respondidas, tendo em vista a escassez da literatura nacional, que se direciona principalmente aos estudantes recém ingressados na universidade, sobretudo aos do curso de Medicina.

Concluímos que há uma alta prevalência total (25\%) de TMM nos estudantes, o que evidencia a necessidade de um projeto político-pedagógico que vise o bem-estar dessa população e promoção da saúde mental, diagnóstico e tratamento precoce.

\section{Agradecimentos}

Pela coleta de dados agradecemos a colaboração da Coordenação e dos Professores dos cursos de Enfermagem da UEMS e da UFMS, e dos cursos de Letras, de Ciência da Computação e de Direito da UEMS. Agradecemos também aos funcionários da Divisão de Recursos Humanos e da Divisão de Assuntos Acadêmicos da UEMS, em especial à Maria Aparecida Dias e Lair Aparecida Cardoso Espíndola, pela disponibilidade em fornecer-nos os dados referentes à comunidade discente e docente. A primeira autora agradece às instituições: UEMS, pela liberação das atividades regulares e UNICAMP, pela oportunidade da continuidade de seu processo de capacitação profissional.

\section{Referências}

Abdo, C. H. N. (1987). Psicoterapia breve: relato inicial acerca de seis anos de experiência com alunos da USP. Revista de Psiquiatria Clínica, 14, 32-35.

Abdo, C. H. N., \& Fortes, J. R. A. (1978). Higiene mental do universitário. Revista de Psiquiatria Clínica, 7, 1-7.

Albuquerque, M. A. (1973). A saúde mental do universitário. Neurobiologia, Suplemento, 36, 1- 12.

Almeida, M. M. G., Souza, M. G., Paiva, M. S., \& Lima, S. A. (1980). Interesse de universitários em aspectos de saúde. Revista Brasileira de Enfermagem, 33, 157-165.

Almeida Filho, N. A., Mari, J. J., Coutinho, E., França, J. F., Fernandes, G., Andreoli, S.B., \& Busnello, E. D’A. (1992). Estudo multicêntrico de morbidade psiquiátrica em áreas urbanas brasileiras (Brasília, São Paulo, Porto Alegre). Revista da Associação Brasileira de Psiquiatria, 14, 93-104.

Baker, R. W., \& Nidorf, L. J. (1964). Pattern of occurrence of psychological disturbances in college students as a function of years level. Journal of Clinical Psychology, 20, 530-531.

Benvegnú, L. A., Deitos, F., \& Copette, F. R. (1996). Problemas psiquiátricos menores em estudantes de medicina da Universidade Federal de Santa Maria, RS, Brasil. Revista de Psiquiatria do Rio Grande do Sul, 18, 229-233.

Borini, P., Oliveira, C. M., Martins, M. G., \& Guimarães, R. C. (1994). Padrão de uso de bebidas alcoólicas de estudantes de medicina (Marília, São Paulo). Parte 1. Jornal Brasileiro de Psiquiatria, 43, 93-103.

Brown, B. M. (1978). Depressed college students and tricyclic anti-depressant therapy. The Journal of the American College Health Association, 27, 79-83.

Cavalcanti, C. T. (1958). Notas sobre o abuso de anfetaminas: seus perigos e prevenção. Neurobiologia, 21, 85-91.

Cianflone, A. R. L., \& Fernandez, J. M. (1993). Algumas características do ensino e aprendizado na Faculdade de Medicina de Ribeirão Preto-
USP. Um estudo junto aos alunos de graduação. Medicina Ribeirão Preto, 26, 228-236.

Coutinho, D. M. (1976). Prevalência de doenças mentais em uma comunidade marginal. Um estudo do Maciel. Dissertação de Mestrado não-publicada, Universidade Federal da Bahia, Salvador.

Coutinho, E. S. F. (1995). Fatores sócio-demográficos e morbidade psiquiátrica menor: homogeneidade ou heterogeneidade de efeitos? Tese de Doutorado não-publicada, Universidade Federal da Bahia, Salvador.

D’Andrea, F. F. (1984). Saúde mental na comunidade universitária. Diálogo Médico, 10, 12-16.

D’Andrea, F. F., \& Almeida, O. M. L. (1988). Crise em estudantes de medicina. Jornal Brasileiro de Psiquiatria, 37, 313-315.

Facundes, V. L. D. (2002). Prevalência de transtornos mentais comuns em estudantes de área de saúde da Universidade de Pernambuco e sua associação com algumas características do processo ensino-aprendizagem. Dissertação de Mestrado não-publicada, Universidade Federal de Pernambuco, Recife.

Facundes, V. L. D., \& Ludermir, A. B. (2005). Transtornos mentais comuns em estudantes da área de saúde. Revista Brasileira de Psiquiatria, 27(3), 194-200.

Fernandez, J. M., \& Rodrigues, C. R. C. (1993). Estudo retrospectivo de uma população de estudantes de medicina atendidos no ambulatório de Clínica Psiquiátrica do Hospital das Clínicas da Faculdade de Medicina de Ribeirão Preto. Medicina Ribeirão Preto, 26, 258-269.

Fortes, J. R. A. (1972). Saúde mental do universitário. Revista da Associação Médica Brasileira, 18, 463-466.

Fortes, J. R. A., \& Abdo, C. H. N. (1981). Saúde mental do estudante. Revista da Associação Médica Brasileira, 7, 369-372.

Frank, H. (1979). Psychodynamic conflicts in female law students. The American Journal of Psychoanalysis, 39, 65-69.

Giglio, J. S. (1976). Bem-estar emocional em universitários. Um estudo preliminar. Tese de Doutorado não-publicada, Universidade Estadual de Campinas, Campinas.

Giglio, J. S. (1981). Um estudo de fatores biológicos, sociais e ambientais com provável influência no bem-estar psicológico de universitários. Revista da Associação Brasileira de Psiquiatria, 3, 142-146.

Goldberg, D. P. (1972). The detection of psychiatric illness by questionnaire: a technique for the identification and assessment of non psychotic psychiatric illness. Londres: Oxford University Press.

Goldberg, D., \& Huxley, P. (1992). Common mental disorders: a bio-social model. Londres / Nova York: Tavistock / Routledge.

Grofe, J. (1963). Depression in college students. The Journal of the American College Health Association, 20, 57-60.

Hahn, M. S. (1994). Estudo da clientela de um programa de atenção em saúde mental junto ao estudante universitário de São Carlos. Dissertação de Mestrado não-publicada, Universidade Estadual de Campinas, Campinas.

Hahn, M. S., Ferraz, M. P. T., \& Giglio, J. S. (1999). A saúde mental do estudante universitário: sua história ao longo do Século XX. Revista Brasileira de Educação Médica, 23, 81-89.

Jorge, M. S. (1996). Situações vivenciadas pelos alunos de enfermagem durante o curso, no contexto universitário, apontadas como norteadoras de crises. Revista da Escola de Enfermagem da USP, 30, 138-148.

Jorge, M. S., \& Rodrigues, A. R. F. (1995). Serviços de apoio ao estudante oferecidos pela Escola de Enfermagem no Brasil. Revista Latino-Americana de Enfermagem, 3, 59-68.

Kysar, J. E. (1962). Going steady and depression in college women. The Journal of the College Health Association, 11, 111-118.

Loreto, G. (1958). Sobre problemas de higiene mental dos universitários. Neurobiologia, 21, 274-283.

Loreto, G. (1963). Problemática sexual de universitários do sexo masculino. Neurobiologia, 26, 250-258. 
Loreto, G. (1964). O aconselhamento psicológico como meio para a formação do estudante em Psicologia Médica e em Psiquiatria. Neurobiologia, 27, 221-231.

Loreto, G. (1965a). Contribuição do aconselhamento psicológico para a saúde mental dos universitários. Neurobiologia, 28, 283-296.

Loreto, G. (1965b). Vocação médica. Psicologia do estudante de medicina. Neurobiologia, 28, 71-80.

Loreto, G. (1972). Saúde mental do universitário. Neurobiologia, 35, 253-276.

Loreto, G. (1985). Uma experiência de assistência psicológica e psiquiátrica a estudantes universitários. Tese de Livre Docência não-publicada, Universidade Federal de Pernambuco, Recife.

Lucena, J., \& Loreto, G. (1968). Informação sobre o serviço de higiene mental para estudantes da Faculdade de Medicina da Universidade Federal de Pernambuco. Neurobiologia, 31, 43-50.

Ludermir, A. B. (2000). Inserção produtiva, gênero e saúde mental. Cadernos de Saúde Pública, 16, 647-659.

Mari, J. J. (1987). Psychiatric morbidity in three primary medical care clinics in the city of São Paulo. Issues on the mental health of the urban poor. Social Psychiatry, 22, 129-138.

Millan, L. R. (1997). A saúde mental do estudante de medicina. Revista Brasileira de Medicina Psicossomática, 1, 135-140.

Millan, L. R., Souza, E. N., De Marco, O. L. N., Rossi, E., \& Arruda, P. V. (1998). O I Encontro Paulista dos serviços de assistência psicológica ao estudante universitário. Revista do Hospital das Clínicas, Faculdade de Medicina de São Paulo, 53, 156-161.
Miranda, P. S. C., \& Queiróz, E. A. (1991). Pensamento suicida e tentativa de suicídio entre estudantes de medicina. Revista da Associação Brasileira de Psiquiatria, 13, 157-160.

Niemi, T. (1988). Problems among students seeking mental health care. Journal of American College Health, 36, 353-354.

Noto, J. R. S., Avancine, M. A. T. O., Martins, M. C. F. N., \& Zimmermann, V. B. (2001). Atenção à saúde mental do estudante de medicina. Revista Brasileira de Educação Médica, 25, 71-75.

O’Neil, M. K., \& Marziali, E. (1976). Depression in a university clinic population. Canadian Psychiatric Association Journal, 21, 477-481.

Pasquali, L., Gouveia, V. V., Andriola, W. B., Miranda, F. J., \& Ramos, A. L. M. (1996). Questionário de Saúde Geral de Goldberg: manual técnico QSG. São Paulo: Casa do Psicólogo.

Ribas, J. C. (1975). Abusos de anfetaminas e juventude universitária. Revista de Psiquiatria Clínica, 4, 17-25.

Rimmer, J., Halikas, J. A., \& Schuckit, M. A. (1982). Prevalence and incidence of psychiatric illness in college students: a four year prospective study. Journal American College Health, 30, 207-211.

Rodrigues, A. R. F., Scatena, M. C. M., \& Labate, R. C. (1997). O aluno ingressante na enfermagem. Revista de Enfermagem da UERJ, 5, 331-339.

Santana, V. S. (1978). Estudo epidemiológico das doenças mentais em um bairro de Salvador - nordeste de Amaralina. Dissertação de Mestrado não-publicada, Universidade Federal da Bahia, Salvador.

Spalt, L. (1977). Affective disorders in university student psychiatric patient. The Psychiatric Quarterly, 49, 56-61.

${ }^{1}$ O presente estudo faz parte da Pesquisa em Saúde Mental e Qualidade de Vida em Estudantes Universitários do Doutoramento em Ciências Médicas, Área de Saúde Mental da UNICAMP, da primeira autora, sob orientação do segundo autor.

Ednéia Albino Nunes Cerchiari, doutora em Ciências Médicas (Saúde Mental) pela Universidade Estadual de Campinas, é professora titular na Universidade Estadual de Mato Grosso do Sul e coordenadora do curso de Enfermagem. Endereço para correspondência: Rua Ibirapuera, 240 (Jardim Ibirapuera); Campo Grande, MS; CEP: 79041-290. Tel.: (67) 341-1621.E-mail: edcer@terra.com.br Dorgival Caetano, doutor pela University of Cambridge, é professor titular na Universidade Estadual de Campinas e Diretor Clínico do Instituto de Saúde Mental.

Odival Faccenda, matemático, mestre em Estatística, e doutor em Agronomia pela Universidade Estadual Paulista Júlio de Mesquita Filho, é professor titular na Universidade Estadual de Mato Grosso do Sul e na Universidade Federal de Mato Grosso do Sul. 